\title{
Hemilaminectomy for the removal of the spinal lesions
}

\author{
IS Öktem*,1, H Akdemir ${ }^{1}$, A Kurtsoy ${ }^{1}$, RK Koç ${ }^{1}$, A Menkü ${ }^{1}$ and B Tucer ${ }^{1}$ \\ ${ }^{1}$ Department of Neurosurgery, University of Erciyes, Medical School, Kayseri, Turkey
}

\begin{abstract}
Objective: We evaluated 20 patients with spinal lesions with respect to the value of unilateral hemilaminectomy at the Department of Neurosurgery, Erciyes University, Medical Faculty, Kayseri, Turkey. The operative technique of the limited approach for spinal lesions is described.

Methods: The study is based on 20 prospective consecutive patients with spinal lesions who had unilateral hemilaminectomy. There were 12 women and eight men. Age ranged from 17 to 63 years mean (42 years) with a slight preponderance of women patients $(60 \%)$. Spinal lesions were cervical in three cases, lumbar in five cases, and thoracic in 12 cases. Hemilaminectomy was performed by using a high speed drill.

Results: Postoperative neurological status was unchanged in six cases, improved in 11 cases, and worsened in three cases. We only observed two cases of wound infections that were not related to our surgical approach. At the follow-up evaluation, which occurred approximately 25 months after surgery none of the patients showed spinal deformity or spinal instability.

Conclusion: The rationale of attempting unilateral approach is to avoid damage to the dorsal static structures of the vertebral column. With the precise preoperative definition of the relationship of tumor to the surface of the spinal cord by contrast enhanced MRI, unilateral approaches might be more applicable to spinal lesions except invasive extradural lesions.

Spinal Cord (2000) 38, $92-96$
\end{abstract}

Keywords: hemilaminectomy; spinal lesions; spinal instability

\section{Introduction}

In order to remove spinal tumors, a bilateral laminectomy has usually been recommended. ${ }^{1-3}$ However, clinical ${ }^{1,2,4-9}$ and biomechanical studies ${ }^{10}$ have shown that laminectomy may cause postoperative spinal instability due to the damage of ligaments and intervertebral joints, which may induce kyphosis after many years. Theoretically, the unilateral approach to the spinal canal should help to solve these problems. In order to reduce the risk of post-laminectomy spinal instability and deformity, Raimondi et al. ${ }^{1}$ and Parkinson $^{7}$ recommended osteoblastic laminotomy originally described by Bickham ${ }^{11}$ and Hoffman. ${ }^{12}$ Unilateral hemilaminectomy was reported by Tay$\operatorname{lor}^{13,14}$ and was popularised by Eggert et al. ${ }^{3}$ and Chiou et al. ${ }^{15}$ In this study, 20 patients suffering from spinal lesions were studied with respect to the value of unilateral hemilaminectomy.

\section{Patients and methods}

Twenty prospective consecutive patients with spinal lesions who were operated on via unilateral hemilaminectomy at our institutes between 1992 and 1996 have been reviewed and analyzed. The study consisted

*Correspondence: IS Öktem, M. Kemal Paşa Bulvari, Tarcan Apt. no. 129/21, Kocasinan, Kayseri, Turkey of 12 female and eight male patients with a mean age of 42 years (range 17-63 years). All patients suffered from local or radicular pain and sensory or motor disturbance of the extremities. Preoperative neurological examination showed weakness of the extremities in 18 cases, sensory disturbances in 12 cases, and sphincter dysfunction in seven cases. Since 1992 hemilaminectomy has been used routinely for the removal of all spinal cord tumors with the exception of those extradural tumors which extensively invade local soft tissue structures bilaterally.

In 15 cases, contrast enhanced MRI was used to demonstrate the side, size and location of the suspected tumor (Figure 1a,b). For the diagnosis of the other five cases, myelo-CT was used. Preoperative, early and late postoperative spinal X-rays, CT-scan and MRI were also obtained to evaluate the spinal instability (Figure 3a,b).

All patients were operated on in a prone position. If level determination was needed, direct X-ray was undertaken intraoperatively. A longitudinal midline incision was performed. According to the details of preoperative radiological findings, the resection of bone and ligaments is restricted to the side of the tumor. Hemilaminectomy was carried out using an intraoperative microscope and high speed drill. The vertebral arch was drilled from medial to lateral and 
a

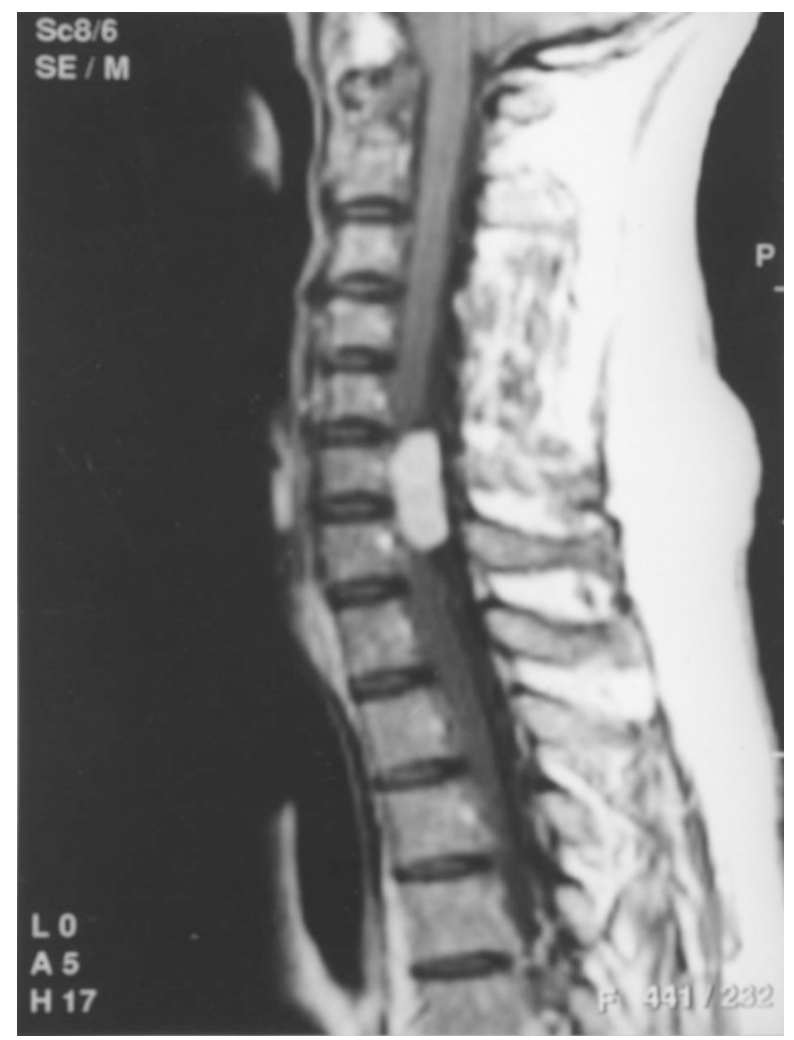

b

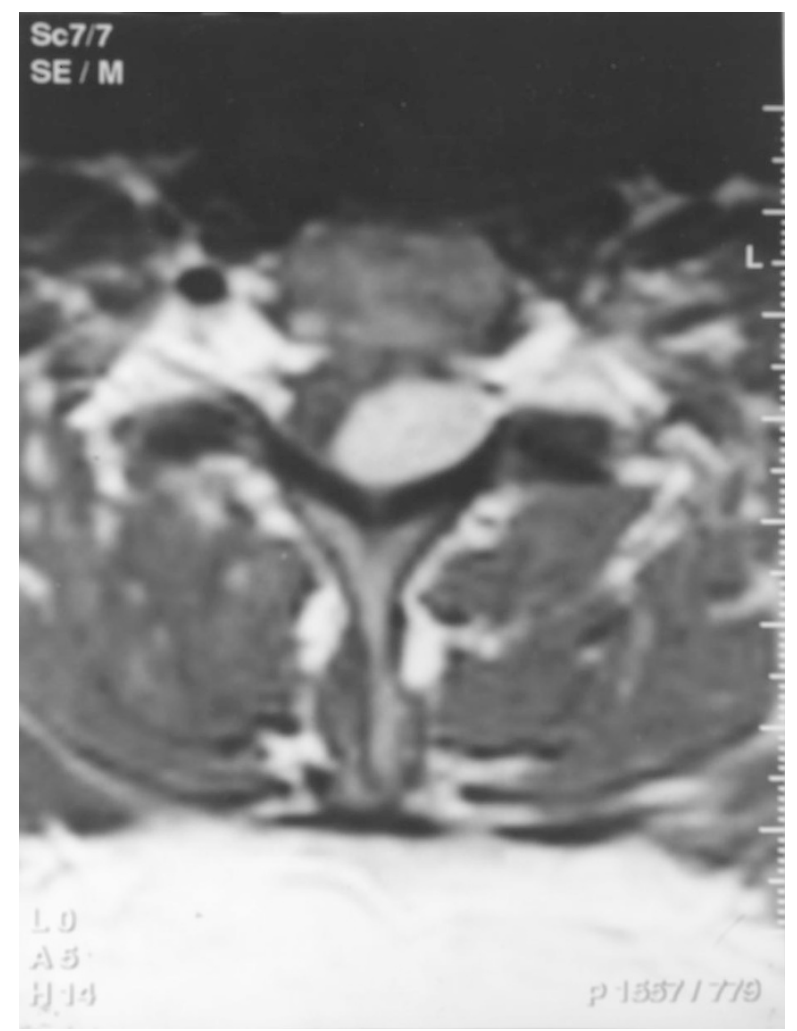

Figure 1a,b $T_{1}$ weighted gadolinium sagittal and axial MRI demonstrates heterogeneously enhancing, schwannoma of the cervical spine

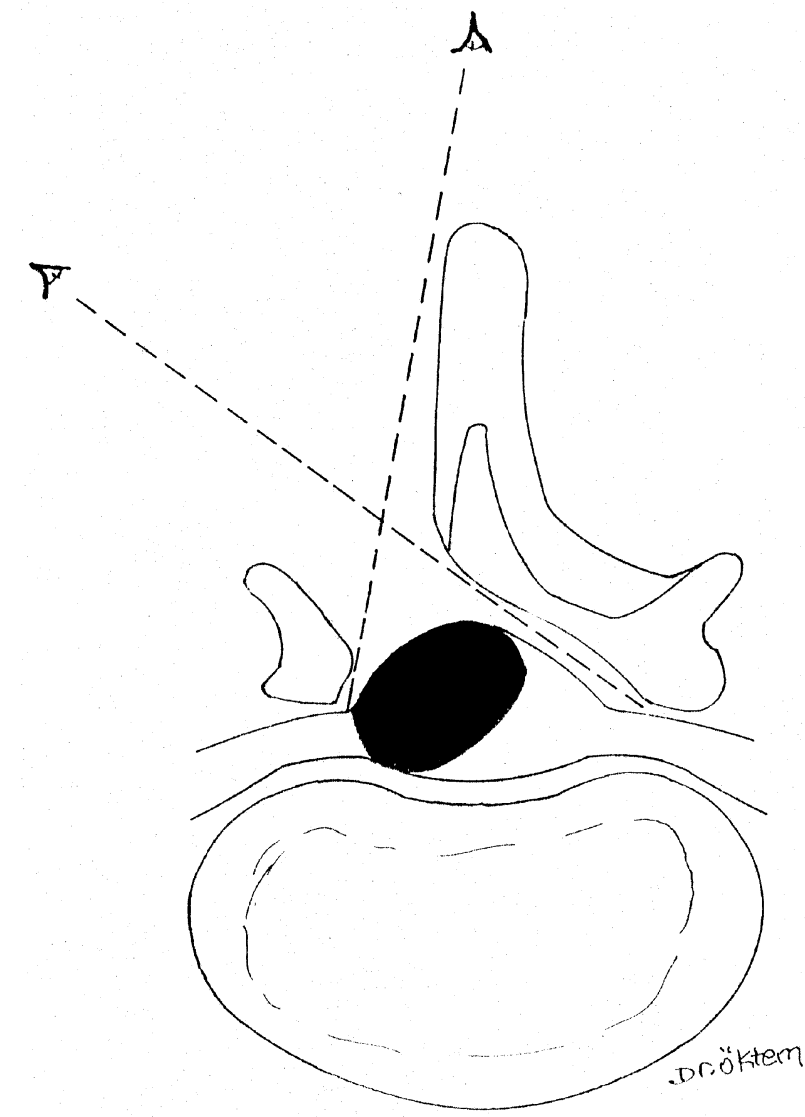

Figure 2 The exposure of the spinal lesion by hemilaminectomy corridor

was continued until ipsilateral root or roots were seen. The ligamentum flavum was removed and the base of the spinous process was also drilled until the contralateral root or dural curve was seen (Figure 2). As necessary, parts of articular process and the pedicle were removed and the dural aspect of the contrlateral lamina was minimally drilled. In intradural lesions, the dura was opened longitudinally at the midline. All patients were carefully observed regarding neurological status, kyphosis and scoliosis after the postoperative period.

\section{Results}

All patients were given rehabilitation therapy beginning on the second postoperative day, although external orthotics were not used in any patients. Before discharge all patients were re-evaluated according to subjective complaint, neurological deficit and bladder function, compared to their preoperative neurological status. Each category was divided into three grades: improved, unchanged and worsened. Postoperative neurological status was unchanged in six cases, improved in 11, and worsened in three cases (one case of subjective complaint, one case of neurological deficit, and one case of sphincter disturbance). Spinal lesions were 
located in the cervical area in three cases, in the lumbar region in five cases, and in the thoracic regions in 12 cases. These cases included 16 extramedullary, three extradural, and one intramedullary lesions. The location and histopathological findings of our cases are shown in Table 1

Steroids were not used. Postoperative pain was relieved by narcotic analgesics on the first day and

a

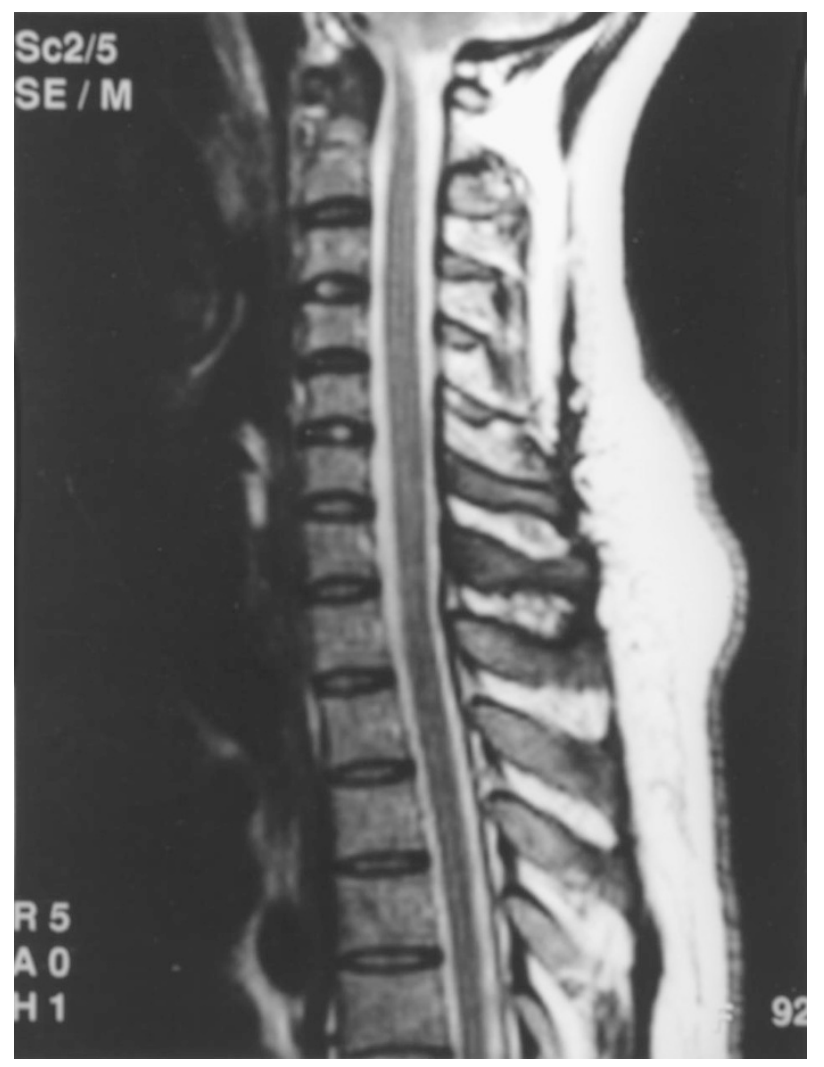

b

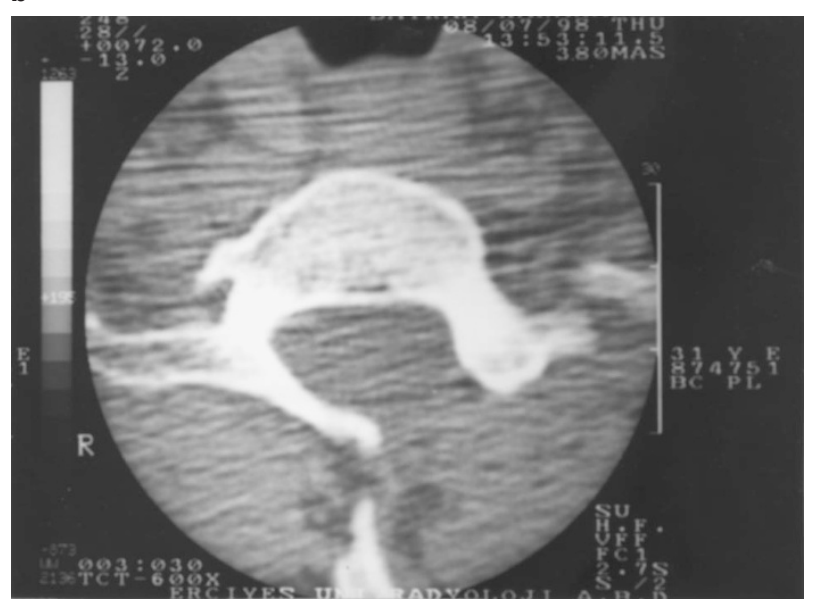

Figure 3a,b Postoperatively MRI and CT scan demonstrate the tumor resection and hemilaminectomy with normal vertebral column alignment and stability then by nonsteroidal anti-inflammatory drugs. In two cases there was wound infection which improved after antibiotic therapy.

There were no other complications. One patient suffering from extradural tumor died during the hospitalisation due to cardiac infarction. In most of the cases, lesions were exposed with two or three levels hemilaminectomy and in one case, five levels (T9-LI) hemilaminectomy was needed for a dural A-V malformation.

The operative approach had to be changed from unilateral to bilateral in three cases. In these three subtotally resected tumors, the problem was the invasiveness of the tumors, not the chosen surgical approach. We did not observe any disadvantage of hemilaminectomy. At follow-up evaluation none of the patients showed kyphosis or scoliosis in the postoperative period (mean 25 months).

\section{Discussion}

Although conventional laminectomy continues to be commonly used for spinal cord tumors, it may be associated with a number of postoperative complications. These problems, which include spinal deformity, instability, epidural fibrosis and the absence of osseous protection for the spinal cord, may lead to a progressive myelopathy. ${ }^{9,16-20}$ Post-laminectomy kyphoscoliosis is the major problem which may occur within weeks to years after laminectomies for tumor or trauma, ${ }^{1,2,4-8,21}$ especially in children before reaching bone maturity. ${ }^{2,48}$ Kyphosis is commonly associated with instability and results in an anterior compression of the spinal cord or nerve roots. ${ }^{21-23}$ Musculoligamentous insufficiency related to surgical stripping and denervation of the posterior paraspinal muscle complex has been suggested to be responsible for these progressive post-laminectomy problems. ${ }^{24,25}$ Yasuoka et $a .^{2}$ proposed that the visco-elasticity of the remaining ligaments after laminectomy might be another cause for postoperative spinal deformities. In addition to the age at operation, the site of laminectomy is also an important factor. In an effort to rate post-laminectomy deformity, a number of different operative approaches have been proposed. ${ }^{3,15,24,25}$ Inoue et $a .^{26}$ reported that the mechanism of spinal deformity after surgical removal of a cervical spinal cord tumor or tumorous lesions was studied in 36 patients, in which they compared laminectomy and laminoplasty groups. In the laminectomy group, kyphosis of the upper cervical spine and compensatory increased lordosis of the lower cervical spine were observed in the $\mathrm{C} 2$ laminectomy patients. Localised kyphosis of the spine at the cervicothoracic junction and compensatory increased lordosis of the upper cervical vertebrae were noted in the C7 laminectomy patients. In the laminoplasty group, spinal deformities were less frequently observed, and when present, the deformity was limited to a slight increase of lordosis, even in patients who 
Table 1 Location and histopathology of the lesions

\begin{tabular}{llll}
\hline & Intramedullary & Extramedullary & Extradural \\
\hline Cervical & Astrocytoma (1) & Meningioma (2) & Tuberculoma (1) \\
Thoracic & - & Meningioma (5) & Turinoma (4) \\
& & A-V malformation (1) & \\
& & Subdural empyema (1) & Osteoblastoma (1) \\
Lumbosacral & - & Meningioma (1) & Sarcoma (1) \\
& & Neurinoma (1) & 3 \\
\hline
\end{tabular}

had the facetectomy. Gros et $a .^{24}$ describe their conservative lumbar laminectomy in which the laminectomy is performed after the spinous processes have been cut at their base and retracted en bloc laterally. Parkinson ${ }^{7}$ and Raimondi et al. ${ }^{1}$ reviewed the method of osteoblastic laminotomy originally described by Bickham ${ }^{11}$ and Hoffman. ${ }^{12}$ However, this technique is said to be quite difficult and time consuming. ${ }^{1,7}$ The rationale for more limited approaches to the vertebral column than laminectomy is to preserve the structures, important in spinal stability. The unilateral approach by hemilaminectomy completely avoids damage to the interspinous ligaments, intervertebral joints and paravertebral muscles of the opposite side. By this means the dorsal static structures of the vertebral column are injured less than by a conventional laminectomy or by an osteoblastic laminectomy. On the other hand, Guidetti criticised the high risk of injury to the spinal cord when unilateral approaches are used. ${ }^{27}$ Exposure of the dorsal parts of the spinal cord after hemilaminectomy is equivalent to that after laminectomy (Figure 2). Our experience indicates that hemilaminectomy is useful for the removal of any kind of spinal lesion with the prerequisite of exact preoperative definition of the tumor location. We did not observe any disadvantage of hemilaminectomy in our cases. If, during the operation, the surgeon feels unable to remove the tumor by hemilaminectomy without damage to the spinal cord, the unilateral approach can be changed into laminectomy during operation. In the present study, only three patients hemilaminectomy was extended to a complete laminectomy during the operation. In these three cases, tumors were resected subtotally because of the invasive extradural tumors.

Chiou et al. ${ }^{15}$ reported their experience in 256 spinal tumors which were removed by using unilateral or bilateral hemilaminectomy. They compared these two approaches and concluded that the unilateral approach can be applied to extradural tumors without disadvantage and in intradural and extrameduller tumors it was seen to be even superior to bilateral laminectomy. Sarioğlu et al. ${ }^{28}$ reported their experience in 40 spinal tumors which were removed by using unilateral hemilaminectomy. They concluded that the unilateral hemilaminectomy can be applied to all spinal tumors except bilaterally extensive invading extradural tumors with the aid of microneurosurgical technique.

With the development of MRI, it is possible to demonstrate shape, size, and relations to the cord precisely. With the precise preoperative definition of the relationship of tumor to the surface of the spinal cord by contrast enhanced MRI unilateral approaches might be more often applicable for spinal tumors. MRI and thus microsurgical techniques allow us to reach and remove spinal tumors by unilateral approach. In the surgery of the spinal lesions our goal was to obtain adequate exposure of the lesion, to remove the lesion totally without injury to the spinal cord and nerve roots, and to preserve spinal column stability. In all cases but three, it was possible to remove the tumor totally in the present study. Our series of these lesions is small and the follow-up period is short to make a definitive statement with respect to the applicability of unilateral approaches to all spinal lesions.

\section{References}

1 Raimondi AJ, Guiterrez FA, Rocco CD. Laminotomy and total reconstruction of the posterior spinal arch for spinal canal surgery in childhood. $J$ Neurosurg 1976; 45: 555-560.

2 Yasuoka S, Hamlet AP, Laws ER, MacCarty CS. Pathogenesis and prophylaxis of postlaminectomy deformity of spine after multiple level laminectomy: Difference between children and adults. Neurosurgery 1981; 9: $145-152$

3 Eggert HR, Scheremet R, Seeger W, Gaitzsch J. Unilateral microsurgical approaches to extramedullary spinal tumors. Operative technique and results. Acta Neurochir 1983; 67: $245-$ 253.

4 Alexander JRE. Postlaminectomy kyphosis. In: Wilkins RH, Rengachary SS (eds). Neurosurgery. McGraw Hill: New York 1985 , pp 2293-2297.

5 Bradford DS. Spinal instability: Orthopedic perspective and prevention. Clin Neurosurg 1980; 27: 591-610.

6 Hendrick EB. Spinal cord tumors in children. In: Youmans JR (ed). Neurological Surgery. 5. WB Saunders, Philadelphia: London 1982, pp 3215-3221.

7 Parkinson D. Replacement laminotomy. Surg Neurol 1977; 8: $277-279$.

8 Reimer R, Onofrio BM. Astrocytomas of the spinal cord in children and adolescents. $J$ Neurosurg 1985; 63: 669-675.

9 Cattel HS, Clark GL. Cervical kyphosis and instability following multiple laminectomies in children. J Bone Joint Surg 1967; 49-A: $713-720$ 
10 Panjabi MM, White III AA. Basic biomechanics of the spine. Neurosurgery 1980; 7: 76-93.

11 Bickham WS. Technique of exposure of the spinal cord and canal: osteoplastic resection and laminectomy. Ann Surg 1905; 41: $372-398$.

12 Hofmann C. Eine einfache Art der temporaren laminektomie. Zbl Chir 1910; 37: 706-707.

13 Taylor AS. Unilateral laminectomy. J Neuro Ment Dis 1910; 39: 257.

14 Taylor AS. Hemilaminectomy. Bull NY Acad Med 1927; 3: 24.

15 Chiou SM, Eggert HR, Laborde G, Seeger W. Microsurgical unilateral approaches for spinal tumour surgery. Acta Neurochir 1989; 100: $127-133$

16 Callahan RA et al. Cervical facet fusion for control of instability following laminectomy. J Bone Joint Surg 1977; 59: 991 - 1002.

17 Lonstein JE. Post-laminectomy kyphosis. Clin Orthop 1977; 128: $93-100$.

18 Mikawa Y, Shikata J, Yamamuro T. Spinal deformity and instability after multilevel cervical laminectomy. Spine 1987; 12: $6-11$.

19 Yasuoka S, Peterson HA, MacCarty CS. Incidence of spinal column deformity after multilevel laminectomy in children and adults. J Neurosurg 1982; 57: $441-445$.

20 Ohmori K, Ishida Y, Suzuki K. Suspension laminotomy; a new surgical technique for compression myelopathy. Neurosurgery 1987; 21: $950-957$.
21 Guidetti B, Fortuna A. Long-term results of surgical treatment of myelopathy due to cervical spondylosis. J Neurosurg 1969; 30: $714-721$.

22 Gore DR, Sepic SB, Gardner GM. Neck pain: a long term followup of 205 patients. Spine 1987; 12: $1-5$.

23 Gregorius FK, Estrin T, Crandall PH. Cervical spondylotic radiculopathy and myelopathy. A long term follow-up study. Arch Neurol 1976; 33: 618-625.

24 Gros C et al. Laminectomies conservatrices lombaires. Technique et resultats. Neurochirurgie 1983; 29: 207-209.

25 Yasargil MG, Tranmer BI, Adamson TE, Roth P. Unilateral partial hemilaminectomy for the removal of extra- and intramedullary tumors and AVMs. In: Symon L (ed). Advances and Technical Standards in Neurosurgery. 18, Springer, Wien: New York 1991, pp 113-132.

26 Inoue A, Ikata T, Katoh S. Spinal deformity following surgery for spinal cord tumors and tumorous lesions. Spinal Cord 1996; 34: $536-542$

27 Guidetti B. Removal of extramedullary benign spinal cord tumours. In: Krayenbühl H, Brihaye J, Loew F (eds). Advances and Technical Standards in Neurosurgery. Vol 1, Springer, Wien: New York 1974, pp 173-197.

28 Sarioğlu AÇ et al. Unilateral hemilaminectomy for the removal of the spinal space-occupying lesions. Minim Invas Neurosurg 1997; 40: $74-77$. 